\title{
ANALGESIA AND ANAESTHESIA IN MODERN OBSTETRICS
}

\author{
By G. C. STEeL, M.R.C.S., L.R.C.P., D.A. \\ Anaesthetist, Queen Charlotte's Maternity Hospital, Chelsea Hospital for Women, etc.
}

' Quot homines, tot sententiae.' Many speakers are discussing the relief of pain in midwifery; each has his own hobby-horse. Such a state of affairs proclaims the inescapable fact that, so far, we have failed to make childbirth safely painless. Yet definite progress has been made.

There are many ways in which the problem may be tackled ; there is a wide variety of drugs available, many of which are reasonably safe and effective. It is the lack of understanding of when and how to use them rather than the excessive use of them which is a matter for reproach at the present time.

Every analgesic drug used in midwifery should fulfill as many as possible of the following criteria -it should be effective, safe, easy to administer, cheap and transportable ; it must have a minimal toxic effect on mother and child, and should not affect uterine contractility.

The choice of drug will then be influenced by :-

(a) The action of the drug ;

(b) The needs of the patient ;

(c) The rate of breakdown of the drug.

(a) Drugs used for the alleviation of the pain of childbirth are either sedative, hypnotic, soporific, antispasmodic, analgesic or anaesthetic ; many have a mixed action. It is essential that these properties be carefully assessed and the correct drug given as indicated by the patient's needs. It is as useless to give chloral and potassium bromide in pharmacological dosage to a woman suffering agonizing pain as it is to give her a caudal block in the early stages of labour when she is suffering no pain but is in a state of uncontrollable fright. We must face the fact that, as yet, there is no one drug that is universally efficacious.

\section{(b) The needs of the patient}

No two patients are identical and their needs are correspondingly variable. The wide range of these needs must always be recognized, for failure to do so will result in failure to provide relief. In addition, whatever the mother may need in the form either of reassurance, sedation, analgesia or anaesthesia, it is of the greatest importance that her sleep requirements be not overlooked.

'For some must watch while some must sleep.' It is imperative that no mother should be allowed to lose her reserves of strength through not being adequately rested during the course of a long labour. There is no excuse for the primipara who starts off in the evening with vague and uncertain pains being allowed to lie sleepless through the night so that, with the coming of day, she faces a severe physical and mental ordeal, weary and apprehensive instead of being calm and refreshed. That this should happen is an adverse criticism of the management of the case. A strategic conservation of her strength for the moment when it is most needed should be the aim of such sedation.

\section{(c) The rate of breakdown of the drug}

Once it is realized that this factor should have a strong controlling influence on the choice of drug, then the chances of a baby being born with its vital centres depressed by narcotics is minimized. This applies not only to the analgesic drugs of the first stage, but also to the inhalational agents used later on. At all times, with the normal or abnormal cases, the guiding principle of timing and dosage of drugs should be, that the child must not be handicapped in the first critical minutes of its independent existence by having in its system an undue amount of a depressant drug which it has to break down without the help of the maternal system, from which it has been so recently severed.

Before the various methods are discussed, there is one more aspect of the problem which we must examine, namely the antenatal instruction of the patient in the part that she has to play in the birth of her child and the effect that the analgesia will have on her appreciation of pain. The choice is clear ; one can either give the mother a carefullyworded description of the task that lies ahead of her, emphasizing the work that she has to do rather than the pain that she may feel; with the result that she will be able to co-operate as an intelligent and informed partner, with the analgesic drugs exercising their optimum effect; or one may leave her in total ignorance of the procedure and, during labour, give her drugs, partly hypnotic and all depressant, thus reducing her to the status of an unskilled labourer.

The influence of fear upon the appreciation of pain and the measures that can be taken to combat the fear-tension-pain cycle have been repeatedly emphasized by Grantly Dick Read. Stripped to its essentials it amounts to this : fear begets tension which, in its turn, causes pain. 
This build-up may therefore be best attacked along the following lines: fear is to be eliminated by antenatal instruction and tension by means of suggestion and relaxation exercises. Should this therapy prove so successful as to eliminate the necessity for analgesic drugs, so much the better : if not gas and air or something similar can be used with probably enhanced effect.

It is a great pity that there have not been more attempts to make large scale assessments of the value of this method. Unfortunately, however, it seems that the question of suggestion or indeed of any similar form of therapy in midwifery is doomed all too frequently to arouse the emotional rather than the objective approach in the minds of those concerned.

The advisability of showing the expectant mother how to use the gas and air apparatus during the latter part of her pregnancy has been emphasized by Minnit (1947). Viewed objectively it is hard to find any valid reason for this practice not being generally adopted. Provided that the patient is not psychologically unsuitable; the use of the apparatus. should be carefully demonstrated to her in the antenatal period. The advantages of doing so are that she will, in the first instance, have the reassurance that something will be done to help her. Secondly, that it is easier for her to appreciate what she is being told at this time rather than at the last minute when she is disturbed by her pains and by the bustle of the labour ward.

From the point of view of efficiency, the merits of antenatal instruction are obvious. There remain traces, however, of a curious belief in some obscure ethical reason for a mother going into labour uninstructed in the use of analgesia and in what she will have to do. This is as untenable as the idea that the boxer who goes into the ring untrained and uninstructed is the spiritual better of his well-prepared opponent.

\section{The Conduct of the First Stage}

During the first stage, especially in primipara, the mother is conscious of colicky contractions of a progressive nature. Whilst the initial contractions do not usually register on the consciousness as an overwhelming agony, nevertheless as time goes on and their intensity increases, the inevitability of the process may cause alarm and starts to sap the mother's morale. It is at this time that the value of antenatal training should be manifest. If some form of drug therapy is necessary, those suitable may be classified as :-

\section{Given by mouth}

'Mother's Mist' is a chloral, potassium bromide and tincture of opium mixture, the exact proportions of the three ingredients varying in different hospitals. Given early in labour to an apprehensive woman it may exert a moderate sedative effect; in pharmacological dosage it cannot be expected to have any genuine analgesic effect on the pains of a fully established labour.

The barbiturate group is a very large one, ranging from the lighter members, which are rapidly broken down, to the heavy, which have' a prolonged effect, being slowly broken down. Their effect, generally speaking, is partly sedative, partly hypnotic and partly analgesic. Several of the more rapidly excreted barbiturates have been given during labour in order to produce partial analgesia or partial amnesia. Provided that the rate of breakdown of the drug is always used as a yardstick, the use of the barbiturates for this purpose is justified. Pentothal sodium, pentothal acid and seconal have all been used successfully. Their disadvantage is that it is difficult to assess the rather variable degree of breakdown, and repeat doses are required at frequent intervals. There is no doubt, however, that in a suitable case, the efficacy of gas and air is very considerably enhanced by the use of one of the light barbiturates, though pethidine now seems likely to replace them.

The medium and heavy groups should be used with caution. Generally speaking it is inadvisable to use them for prolonged analgesia towards the end of the first stage. Their place in midwifery is to give the patient who is just starting off with uncertain pains the benefit of a good night's rest. The benefit that accrues from a good night's rest at the beginning of labour cannot be overemphasized. Not only does it conserve the mother's muscular strength for the moment of need, but also tends to prevent her morale from breaking down. To be of safe benefit the drug should be used early on in labour and in adequate dosage ; it is useless and dangerous to let the hours pass by and then give a half dose towards the end of the first stage. As patients show a marked variation in their reactions to the barbiturates, it is well to exclude the possibility of untoward reaction by giving the mother, about a fortnight before the confinement is due, a normal dose of the particular one it is proposed to use.

\section{By injection}

With the advent of the more modern drugs, the use of morphia and scopolamine to produce 'twilight sleep' can no longer be considered justifiable. For morphia, or omnopon and scopolamine, there is, however, still a definite place in midwifery. In cases of primary uterine inertia where sedation is indicated, the drug of choice is morphia or omnopon. The addition of scopolamine is favoured by many. The useless contractions which occur during primary uterine 
inertia are often more painful than those of normal labour. Given this form of treatment, the patient will fall asleep, to awaken several hours later with the cervix well dilated. Once again it is necessary to emphasize that if the patient is to benefit without running undue risk, the drug must be given as early as possible. The full effect of the morphia should never be allowed to overlap into the second stage.

\section{Pethidine}

There can be no doubt that pethidine is gaining a well-deserved place in midwifery. Being both analgesic and antispasmodic, it is especially useful for combating the colicky pain of the first stage. The initial dose should be given as soon as the pains are beginning to cause genuine discomfort, that is, at the time when sedatives such as ' mother's mist' are no longer effective. Generally speaking roo mgm. is sufficient, but some favour an initial dose of $150 \mathrm{mgm}$. A second injection of $100 \mathrm{mgm}$. may be given if necessary, but further doses should not be given without due consideration. Most workers believe that it has little or no adverse effect on the baby, though Gallen and Prescott (1944) feel that it should not be given within $2 \frac{3}{4}$ hours of the estimated time of delivery.

The effect on the strength and frequency of the pains is variable and generally speaking the first or second injection seem to make little difference ; Barnes (1947) noted that in 67 per cent. the contractions were unaffected, in 23.3 per cent. the strength was increased and in 8.8 per cent. decreased. On several occasions the writer has noticed that the pains have died away for a short interval after the third or fourth injection, though not after the first or second. The injection is usually given intramuscularly. In urgent cases it may be given intravenously provided that it is given very slowly.

\section{Pethidine and Scopolamine}

More recently pethidine has been reinforced by the addition of scopolamine. The combination is both rational and efficacious. Pethidine is a marked analgesic, it also has an antispasmodic and, to a certain extent, a sedative action ; to this is added the amnesic and antispasmodic effect of scopolamine. In a series reported by Roberts (1948) the degree of relief obtained was assessed as good in no less than 82 per cent. of cases. Very few cases showed any scopolamine excitement and it is suggested that due regard should be paid to the patient's temperament. Occasionally the combination is repeated, but a large percentage do well on only one dose, or one dose with pethidine only repeated an hour later. This is in interesting contrast to the twilight sleep technique in which the scopolamine, not the morphine, was repeated.

\section{By Inhalation}

The latter part of the first stage is frequently thought to be the most painful part of labour. In addition to the physical pain, the mother has not yet experienced that type of pain which by its very nature proclaims that the end is in sight, and gives her the reassurance of that knowledge. Unfortunately, the obstetrical division of labour into first and second stages tends to create the assumption that the beginning of the second stage is the time for stepping up the degree of analgesia. This is not so: the best time to start increasing the degree of analgesia is in the last half hour of the first stage. It is at this time that the administration of the inhalational agents should be started.

Gas and air is sometimes criticized as being totally inadequate for obstetrical analgesia. Whilst it is true that it is sometimes insufficient for the actual birth, to condemn it as useless shows up the weakness of the nursing and obstetrical staff rather than the defects of the drug. The degree of success obtained with gas and air is in direct proportion to the amount of trouble taken by the attendant. At risk of being monotonous, a few cardinal rules must be reiterated. The patient must be thoroughly and painstakingly instructed in the use of the apparatus as early in labour as possible, or better in the antenatal period. She should be told, firstly, that the gas will not send her to sleep but that it will 'blanket down' the pain very effectively ; secondly, that the fact that she will be able to hear what is being said to her need not make her think that it is not working; finally, so that she can get the full effect of the gas, she should not wait until the contraction is fully established but must start to inhale the moment that she gets the first warning of the approaching pain. Too much attention cannot be paid to the efficient fit of the facepiece and the stopping up of the safety hole.

Lately some new types of gas and air machines have been brought out. The principle is that of the venturi tube in which a gas is passed through a tapered jet at high velocity. The negative pressure which this creates in a side tube attached to the jet sucks in the requisite amount of air. The writer's impression has been that analgesia is more readily attained with this type than with the models which do not use this principle.

Gas and oxygen is the non-austerity counterpart to gas and air, and as the patient can be given a higher concentration of nitrous oxide without fear of suboxygenation, the results are correspondingly better. Given co-operation on the part of the patient and skill and patience on the part of the 
anaesthetist, this method is exceedingly hard to beat for efficacy, combined with safety. It is a very great pity that economic and other factors preclude the wider employment of this excellent form of analgesia.

\section{Trilene}

Trichlorethylene is a valuable and excellent analgesia for use in obstetrics. The rare case that is unmanageable under gas and air will frequently quieten down under trilene. There is no evidence that trilene either slows labour or causes foetal asphyxia, though very occasionally the baby seems to be rather limp and unenthusiastic. Of the various types of apparatus for self administration, the Freedmans and the trilite inhaler both give a fixed concentration; in the Cyprane and the Siebe-German Hyatt, the concentration can be varied by means of a locking mechanism controlled by the anaesthetist or obstetrician. Both the latter types are useful for the single-handed obstetrician who has to apply forceps or undertake some similar manoeuvre.

The value of trilene lies in the fact that it is a most helpful adjuvant to, or replacement for, gas and air or oxygen when this proves inadequate. At the present time the question of allowing midwives to use trilene is being investigated. Whilst one hopes that this may be possible at some future date, it would be a pity if this meant the lighthearted abandonment of gas and air analgesia. The latter can be of great help in labour, and its failures are by no means invariably the failures of the drug or of the apparatus. Ideally the patient can well be started on gas and air followed by trilene when, or if, the former proves inadequate.

\section{The Conduct of the Second Stage}

From the point of view of analgesia, there is little to be feared in the second stage up to the point of the head dilating the vulva, provided that adequate steps have been taken to deal with the last part of the first stage. If gas and oxygen is being given the patient should be given three breaths of pure gas the moment she suspects the pain is starting, followed by a mixture of 85 per cent. gas and 15 per cent. oxygen until the contraction is firmly established, then told to bear down. Trilene added to gas and oxygen or given by itself is very useful in the second stage. Not being excreted as rapidly as nitrous oxide there is some ' hang-over' between pains so that the patient is pleasantly muzzy. Sometimes gas and oxygen is not quite enough to cover the birth of the child ; in this instance the anaesthetist is better advised to add a trace of trilene or ether rather than to lower the oxygen content and so sub-oxygenate the child on the threshold of its independent existence.
Cyclopropane is also suitable, but should not be given if trilene has been already used.

\section{Caudal Block}

Misapprehensions concerning the uses and effects of caudal block are widespread, so it is as well to recapitulate its salient features. Anaesthetic fluid, injected through the sacro-coccygeal ligament into the sacral canal will lie in the sacral portion of the epidural space. If a sufficient amount is injected it will fill up the canal and then strip its way up the lumbar and thoracic portions of the epidural space. When the top level lies between D12 and D8, all the pain.impulses of the uterine contractions are obliterated. (Above D8 the motor supply is cut off and the contractions disappear.) This block can be prolonged for the entire course of labour by further injections at regular intervals, the needle being left in situ.

There can be no doubt that as far as analgesia is concerned this method is excellent. With few exceptions, the labour is genuinely painless. The cervix also softens appreciably and the perineal floor becomes soft and relaxed. There are, however, certain disadvantages which cannot be overlooked. The landmarks are frequently obscured, rendering the proceeding a difficult one as regards technique ; the dangers of infection and of a calamitous fall in blood pressure though remote $\bar{c}$ are nevertheless present. The forceps rate, especially in primipara, is exceptionally high : this is because the head comes down well to the perineal floor but then stays there. At this stage, when the mother should be actively pushing down, she has not the slightest compulsion to do so, being totally unaware of when her contractions are occurring. Even if she is told to push at the right moment she only does so in a half-hearted way, being unable to appreciate either that a contraction is occurring or that her efforts are in fact causing the head to advance. The lack of tonus of the muscles of the birth canal also means that a high proportion of occipito-posterior cases fail to rotate. These are serious drawbacks and it appears unlikely that this method will become universally applicable. The effect of the block in causing relaxation of the cervix is, however, noteworthy, and it may yet find a place as a form of treatment in appropriate cases.

\section{The Operative Procedures of Obstetrics}

Forceps. The straightforward low forceps extraction is adequately covered by gas and oxygen aided by a small amount of trilene or ether. At all times anoxia is to be avoided, especially if there is any degree of foetal distress.

The high or mid forceps, possibly complicated by foetal distress and by the necessity for internal 
rotation, is a much more difficult problem; it can be one of the most difficult tasks the anaesthetist has to face and must not be lightly dismissed. The needs of the three parties concerned are far from identical and may indeed prove to be contradictory. The obstetrician must have an adequate degree of relaxation for his manoeuvres, the mother should be adequately protected from the shock that may arise from these manoeuvres : yet the fulfilment of these needs must not entail narcosis of the infant's possibly already enfeebled vital centres. To reconcile these factors under general anaesthesia is a task for the skilled and not for the casual anaesthetist. Two safety rules may be profitably observed; firstly, the patient must be sufficiently deep for the application of the forceps and the subsequent traction; secondly, it is a beneficial practice to give the patient nothing but pure oxygen from the moment the head is born until the cord is clamped.

Consideration of the problem of the complicated forceps case inevitably brings forward the claims of spinal and extradural nerve blocks; claims that are well substantiated in ease of manoeuvrability, the cutting off of shock impulses to the mother and the absence of adverse effects on the child. Against this is arrayed the classical antipathy to the use of spinals in labour as well as the routine risks of fall of blood pressure, spinal headache and the possibility of infection. It is probable, however, that the dangers of spinals in midwifery are due more to the inexperience of the casual anaesthetist than to the drug or the fact of pregnancy. A convincing series of complicated forceps cases done under spinal has been reported on by Anderson (1946). It is also interesting to note that spinal block has been used to control certain cases of post partum uterine atony (Hansen, 1943) and that Weintraub, et al., have successfully treated post spinal headache in obstetrical cases by the application of tight abdominal binders.

A single caudal block has the advantages that the solution remains outside the dura and that the risk of headache is minimized; against this is the fact that the extradural block is much more slowly acting than is an intrathecal one so there is an interval of at least 20 minutes before the forceps can be applied. Furthermore, touch is often only partially affected and so it may be necessary to give a light gas and oxygen as well. This should not be taken as proof that the caudal block is a failure, the patient merely requires to be in a light sleep, . the baby is unaffected and the operation can be carried out easily and unhurriedly. The value of these two types of nerve block for obstetrics has not yet been fully realized.

\section{Caesarean Section}

It must be realized that there is no one form of $\frac{3}{2}$ anaesthesia that is ideal for every case of Caesarean section. The factors that are to be taken into consideration are legion, yet one still hears one method or another being extolled as the universal method of choice. The reason for the operation, the mentality of the patient and the type of operation, should always be considered. Apart from the metabolic diseases such as diabetes which must strongly influence premedication and anaesthesia, there is the profound difference between the classical and the lower segment operation as it affects the anaesthetist. When the classical operation is performed, it can be done under gas oxygen and minimal trilene or ether or with cyclopropane, without any undue risk of narcotizing the infant. But the more deliberate lower segment extraction presents a very different problem. The interval of time between the induction of anaesthesia and the delivery of the infant is much longer with consequent build up of anaesthetic drug in the foetal circulation : more relaxation is needed. Finally the patient has to be taken to a deep plane of anaesthesia to permit of a long length of gauze being packed down around the uterus just a few seconds before the child is launched on its in dependent life. The obstetrician who is able to omit this. latter step confers a boon on the anaesthetist and on the child.

Under these circumstances it is surprising that one still sees the statement that gas and oxygen is sufficient for the operation. It must be clearly understood that the lower segment operation, especially in instances where premedication has been withheld, cannat be covered by gas and oxygen alone without an undesirable degree of suboxygenation being necessitated. Ether, trilene or cyclopropane should be added in sufficient quantities adequately to protect the mother until the moment of incising the uterus, and then pure oxygen should be given until the cord is clamped. Thus the child is given as reasonable a start in life as is possible under the circumstances. After the cord is clamped, the mother can be given $\frac{1}{2} \mathrm{gm}$. of pentothal so that she awakes from a pleasant pentothal anaesthetic. The use of curare in Caesarean section has lately received attention (Gray, 1947).

For a long time there has been widespread mistrust of the use of spinal analgesia for Caesarean section. More recently the question has been reopened and a most convincing series has been put forward by Rufus Thomas (1947). The possibility of increased contractile action of the uterus and that of sub-oxygenation of the patient through paralysis of the lower intercostals and the embarrassment of diaphragmatic action by the 
full term uterus must not be overlooked (Mackintosh, I949).

Epidural analgesia has the advantages that there is no need for special positioning of the patient during or after the operation, and that there is no likelihood of post spinal headache. The degree of analgesia achieved is not as complete as in the case of an intrathecal injection and a light gas and oxygen will probably have to be given, as touch is frequently only partially obliterated. In spite of this and the fact that an epidural injection does not produce analgesia for a good 20 minutes, the procedure is well worth while. The patient need only be kept lightly asleep, the baby cries immediately and the empty uterus contracts down firmly. $35-45 \mathrm{cc}$. of nupercaine $1 / 600$ injected between DI2 and LI will usually suffice. A careful watch should always be kept on the blood pressure.

The solution can also be given as a caudal injection. into the sacral canal. There is little difference in effect between this and a lumbar epidural injection except that slightly greater amounts of the analgesic agent may have to be given with the former approach, as the solution probably has to fill up the sacral canal before it starts to strip its way up the lumbar and thoracic portions of the epidural space.

There is no longer any doubt that these three forms of nerve blocks have a claim to serious consideration as well as general anaesthesia and local nerve block. Eventually their position will be evaluated, but meanwhile we can utter the warning that the dangers probably lie in the inexperience of the casual administrator rather than in the drug or the patient.

Finally, the patient's temperament should be taken into consideration. There are those who have only one wish-to know absolutely nothing about the operation: those who are indifferent and those who have a strong desire to be conscious so that they will hear the baby's first cry. These feelings should not be overlooked.

External version brings us to the mention of chloroform. There is no agent which will give the same degree of relaxation of uterine muscle that is brought about by chloroform. The use of this drug for anaesthesia at the end of the second stage, or for forceps, is gradually being superseded, but it still remains a very useful weapon albeit one that has to be used with great caution.

Over a hundred years have passed since James Young Simpson introduced the use of chloroform in obstetrics. Yet a recent survey, 'Maternity in Great Britain,' shows that only about 5 per cent. of those who had their babies at home in the period reviewed (March 3-9, 1946) were given gas and air analgesia. Such figures should not call forth any great degree of self-satisfaction. A complete change of approach to the problem is overdue.

This can only be brought about by more collaboration on the part of the obstetrician, the anaesthetist, the midwife and the practitioner. At the moment each of these is inclined to work along separate lines rather than together. To a certain extent this is due to each viewing the problem from a different angle. For instance, the obstetrician sees analgesia as only one facet of the whole process of parturition, consequently he is often reluctant to allow the anaesthetist a free hand in his attempts to provide analgesia ; whilst the anaesthetist, the nature of whose everyday work inevitably stresses the question of the relief of pain, may feel, for his part, that progress in this direction is needlessly slow.

These differences of viewpoint can only be reconciled by a mutual effort. The anaesthetist can profitably spend more time in the labour wards than has hitherto been his custom, for obstetrical analgesia and anaesthesia demand skill, experience and a thorough knowledge of the physiology of parturition. The obstetrician, on his side should not encourage the still lingering practice of excluding the anaesthetist from the labour wards : to do so will merely ensure that the analgesia service will never be first class.

'Analgesia is the business of the obstetrician' is no more than an oft reiterated and misleading half truth. It is surely the business of the obstetrician, the anaesthetist, the practitioner and the midwife : and finally the business of that often overlooked but very important person who is invariably at the confinement though everyone else may be late-the patient herself.

The pregnant woman, it is well known, is all too often the recipient of whispered superstitions and tales of obstetrical horror. Yet very few institutions take active steps to counteract this nonsense by means of organized instruction during the antenatal period.

Let us hope that more attention will be paid to the active side of the attempt to make childbirth painless, and that in the future women will come to their confinements trained in the part they have to plan and in the apparatus they have to use, and with fear and ignorance replaced by true knowledge.

\section{BIBLIOGRAPHY}

ANDERSON, A. F. J. (1946), Obst. Gyn. Brit. Emp., 53, 347. BARNES, JOSEPHINE (1947), B.M. $., 1,437$. GALLEN, B., PRESCOTT, F.' (1944), B.M.尹., 1, 176. HANSEN, J. L. (1943), Acta Obst. Gyn. Scand., 22, 305. MACINTOSH. R. R. (1949), B.M. F., r, 409 . Maternity in Great Britain (I948), London.

MINNITT, R. J. (1947), ' Gas and Air Analgesia,' London. READ, GRANTLY DICK (1942), 'Revelation of Childbirth,'

ROBERTS, HILDA (1948), B.M.F., 2, 590.

THOMAS, RUFUS (1947), Proc. Roy. Soc. Med., 40, 557.

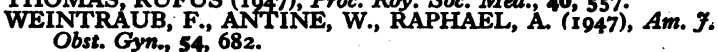

